\title{
Modelagem Molecular de Fármacos
}

\section{Rafael V. C. Guido \& Adriano D. Andricopulo}

A indústria farmacêutica vem passando por grandes transformações guiadas pelas inovações científicas e tecnológicas em seus programas de pesquisa e desenvolvimento (P\&D) de fármacos. Os modelos tradicionais de pesquisa básica estão progressivamente dando lugar a estratégias modernas baseadas em uma combinação de especialidades atuando em caráter multi- e interdisciplinar. Neste contexto, a química medicinal moderna envolve a aplicação de métodos computacionais como a modelagem molecular, que contribuem significativamente para a otimização de propriedades farmacodinâmicas e farmacocinéticas de moléculas bioativas. O presente artigo tem como objetivo apresentar uma abordagem dinâmica de alguns aspectos fundamentais e aplicações da modelagem molecular como ferramenta útil no processo de planejamento de candidatos a novos fármacos.

Palavras-chave: modelagem molecular; planejamento de fármacos; métodos computacionais.

The pharmaceutical industry is facing major challenges on the scientific and technological front of their Research \& Development (R\&D) programs. The modern research paradigm has been and continues to grow at a rapid pace, integrating knowledge of multi- and interdisciplinary teams. Recent advances in medicinal chemistry have created an important foundation in the search for new drug candidates. Modern drug design strategies employ computational methods such as molecular modeling as essential tools in the optimization of pharmacodynamic and pharmacokinetic properties of bioactive molecules. The aim of this chapter is to provide some fundamental concepts and applications of molecular modeling as a useful tool in the design of new drug candidates.

Keywords: molecular modeling; drug design; computational methods. 


\section{Introdução}

\section{QUÍMICA MEDICINAL E A DESCOBERTA DE NOVOS FÁRMACOS}

O processo de descoberta e desenvolvimento de novos fármacos é bastante complexo e fundamenta-se na integração de várias áreas estratégicas, tais como: inovação, conhecimento, tecnologia, gerenciamento e investimentos em Pesquisa e Desenvolvimento (P\&D). ${ }^{1}$ Os avanços da química e biologia, aliados a melhor compreensão de mecanismos fisiológicos e farmacológicos que levam ao aparecimento e desenvolvimento de doenças, tornaram possível o planejamento e a descoberta de fármacos capazes de representar notáveis inovações terapêuticas, proporcionando melhorias significativas na qualidade de vida das diversas populações no mundo. ${ }^{2-4} \mathrm{Na}$ estratégia de planejamento racional de fármacos, os estudos dos processos evolutivos de reconhecimento molecular em sistemas biológicos assumem grande importância, pois constituem as bases fundamentais para a potência, afinidade e seletividade dos fármacos por seus receptoresalvos. A química medicinal possui papel central nesse complexo paradigma de planejamento e otimização de novas moléculas com atividade biológica. ${ }^{5-7}$

A química medicinal possui forte caráter multidisciplinar, abrangendo diversas especialidades importantes, como a química orgânica, bioquímica, farmacologia, informática, biologia molecular e estrutural, entre outras. Segundo a União Internacional de Química Pura e Aplicada (IUPAC, do inglês International Union of Pure and Applied Chemistry), a química medicinal envolve a descoberta, desenvolvimento, identificação e interpretação do mecanismo de ação molecular de compostos biologicamente ativos. Além da descoberta de moléculas bioativas, também estuda os fenômenos envolvidos no metabolismo e o estabelecimento de relações entre a estrutura química e atividade (SAR, do inglês, structureactivity relationships). ${ }^{8}$ A química medicinal é uma ciência interdisciplinar fundamentada em química e que envolve aspectos importantes das ciências farmacêuticas, médicas, biológicas, físicas e computacionais.

\section{FÁRMACOS E INDÚSTRIA FARMACÊUTICA}

$\mathrm{O}$ setor de $\mathrm{P} \& D$ de fármacos é extremamente competitivo e se caracteriza por elevados níveis de investimentos, que estão associados a riscos de proporcional magnitude. A idéia de que as maiores companhias farmacêuticas são as melhores em P\&D tem se tornado um dogma mundial, já que os fármacos da categoria blockbuster (termo do inglês para designar os fármacos com vendas anuais superiores a US\$ 1 bilhão) são os principais responsáveis pelo crescimento da indústria farmacêutica. A capacidade da indústria em destinar recursos para $\mathrm{P} \& \mathrm{D}$ está diretamente relacionada à sua competência em gerar receitas através da venda de um conjunto atrativo de fármacos. ${ }^{9-11}$

$\mathrm{O}$ processo de descoberta e desenvolvimento de novas entidades químicas (NCEs, do inglês, new chemical entities) é longo e envolve altos custos. ${ }^{12}$ Desde a concepção do projeto até a introdução de um único fármaco no mercado farmacêutico, são investidos de 12 a 15 anos em P\&D, com valores totais estimados, no biênio 2004-2006, da ordem de US\$ 500-880 milhões, podendo em alguns casos alcançar cifras superiores a US\$ 1 bilhão (Figura 1). 7,13,14

Inovações terapêuticas de grande sucesso, entretanto, são capazes de gerar rendimentos significativos, na ordem de bilhões de dólares anuais. Dentre os fármacos incluídos na categoria blockbuster, estão o hipolipemiante atorvastatina (Lípitor ${ }^{\circledR}$, da Pfizer), que gerou extraordinários US\$ 12,68 bilhões em vendas somente no ano de 2007; o antitrombótico clopidogrel (Plavix ${ }^{\circledR}$, da Sanofi Aventis); o antiulceroso esomeprazol (Nexium ${ }^{\circledR}$, da AstraZeneca); e o anti-psicótico olanzapina (Zyprexa ${ }^{\circledR}$, da Eli Lilly), entre outros (Tabela 1). ${ }^{15}$

O processo de descoberta e desenvolvimento de fármacos é dividido em duas grandes fases, a saber: (i) pré-clínica (descoberta ou pesquisa básica) e (ii) clínica (desenvolvimento). ${ }^{716}$ Nos estágios iniciais da fase préclínica, as pesquisas se concentram na identificação e otimização de moléculas pequenas capazes de modular a atividade do alvo macromolecular eleito para o processo de planejamento (Figura 2). A validação do alvo molecular selecionado é fundamental, pois estabelece a sua relevância no processo fisiopatológico em estudo, além de evidenciar se a sua modulação seletiva é capaz de gerar a resposta farmacológica esperada no controle de uma doença ou disfunção em humanos. , $^{817,18}$

As moléculas bioativas ou ligantes (do inglês, hits) 


\section{Artigo convidado 2}

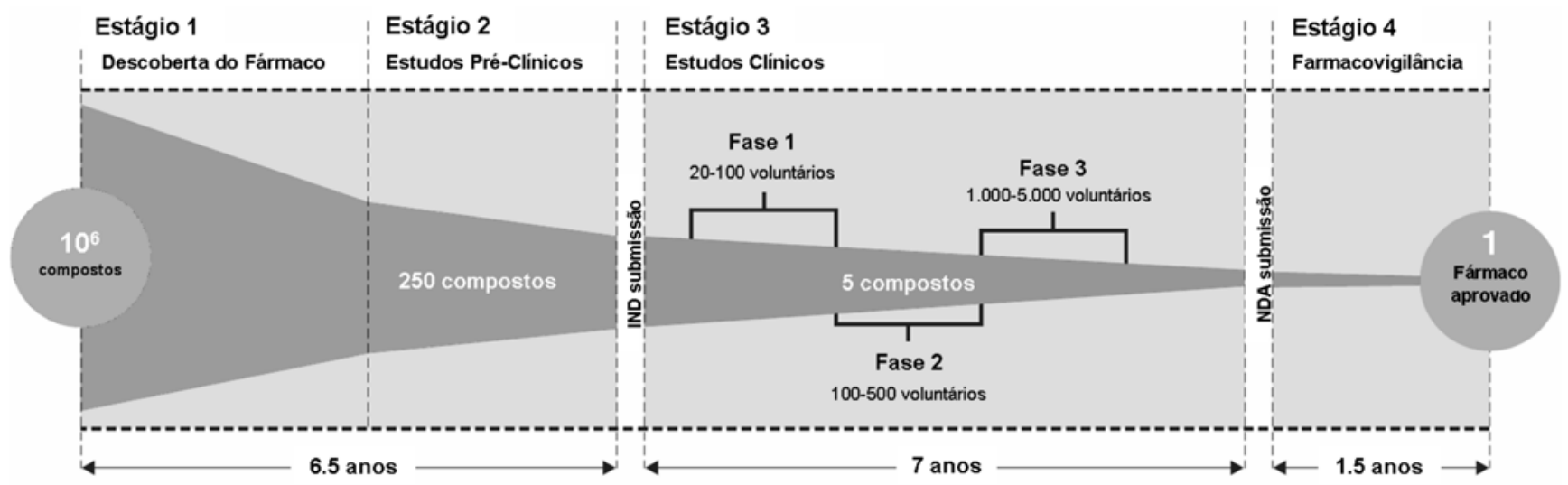

Figura 1: Visão geral do processo de $\mathrm{P} \& \mathrm{D}$ de um novo fármaco. ${ }^{14}$

tem sua origem a partir de produtos naturais ou através de síntese orgânica ou coleções combinatórias (Figura 2). As moléculas bioativas podem ser identificadas através de triagens reais ou virtuais, ou ainda através de planejamento racional, mas em todos os casos as suas propriedades biológicas devem ser determinadas experimentalmente. Nessa fase inicial geralmente são identificadas moléculas com baixa afinidade, que necessitam ser otimizadas em relação a uma série de propriedades (e.g., potência, afinidade, seletividade, biodisponibilidade, toxidez). Os compostos bioativos com melhores propriedades são selecionados como compostos líderes (do inglês, lead compounds) para posterior otimização molecular (Figura 2)..$^{17,20,21}$

Tabela 1: Os 10 medicamentos da categoria blockbuster mais vendidos no ano de $2007 .{ }^{15}$

\begin{tabular}{|c|c|c|c|c|c|}
\hline Posição & Medicamento & $\begin{array}{c}\text { Classe } \\
\text { terapêutica }\end{array}$ & Indicação & Companhia & $\begin{array}{c}\text { Vendas em 2007 } \\
\text { (USS bilhões) }\end{array}$ \\
\hline 1 & $\begin{array}{c}\text { Lipitor }^{8} \\
\text { (atorvastatina) }\end{array}$ & $\begin{array}{l}\text { Redutor de } \\
\text { colesterol }\end{array}$ & & Pfizer & 12,68 \\
\hline 2 & $\begin{array}{c}\text { Plavix }^{*} \\
\text { (clopidrogel) }\end{array}$ & Anticoagulante & $\begin{array}{l}\text { Doenças } \\
\text { cardiacas }\end{array}$ & $\begin{array}{l}\text { Sanofi- } \\
\text { Aventis }\end{array}$ & 8,77 \\
\hline 3 & $\begin{array}{c}\text { Advair }^{\star 2} \\
\text { (fluticasona; salmeterol) }\end{array}$ & $\begin{array}{l}\text { Anti-inflamatório; } \\
\text { broncodilatador }\end{array}$ & Asma & GSK & 6,98 \\
\hline 4 & $\begin{array}{c}\text { Nexium }^{\circ} \\
\text { (esomeprazol) }\end{array}$ & Anti-úlcera & Úlcera & AstraZeneca & 5,22 \\
\hline 5 & $\begin{array}{c}\text { Diovan }^{*} \\
\text { (valsartan) }\end{array}$ & Anti-hipertensivo & $\begin{array}{l}\text { Hipertensão } \\
\text { arterial }\end{array}$ & Novartis & 5,01 \\
\hline 6 & $\begin{array}{c}\text { Zyprexa }^{8} \\
\text { (olanzapina) }\end{array}$ & Anti-psicótico & Esquizofrenia & Eli Lilly & 4,76 \\
\hline 7 & $\begin{array}{c}\text { Singulair }^{8} \\
\text { (montelucaste) }\end{array}$ & Broncodilatador & Asma & Merck \& Co & 4,27 \\
\hline 8 & $\begin{array}{r}\text { Seroquel}^{8} \\
\text { (quetiapina) }\end{array}$ & Neuroléptico & Esquizofrenia & AstraZeneca & 4,03 \\
\hline 9 & $\begin{array}{c}\text { Effexor }^{8} \\
\text { (venlafaxina) }\end{array}$ & Anti-depressivo & Depressão & Wyeth & 3,80 \\
\hline 10 & $\begin{array}{r}\text { Cozaar }^{8} \\
\text { (lozartan) }\end{array}$ & Anti-hipertensivo & $\begin{array}{l}\text { Hipertensão } \\
\text { arterial }\end{array}$ & Merck \& Co & 3,35 \\
\hline
\end{tabular}




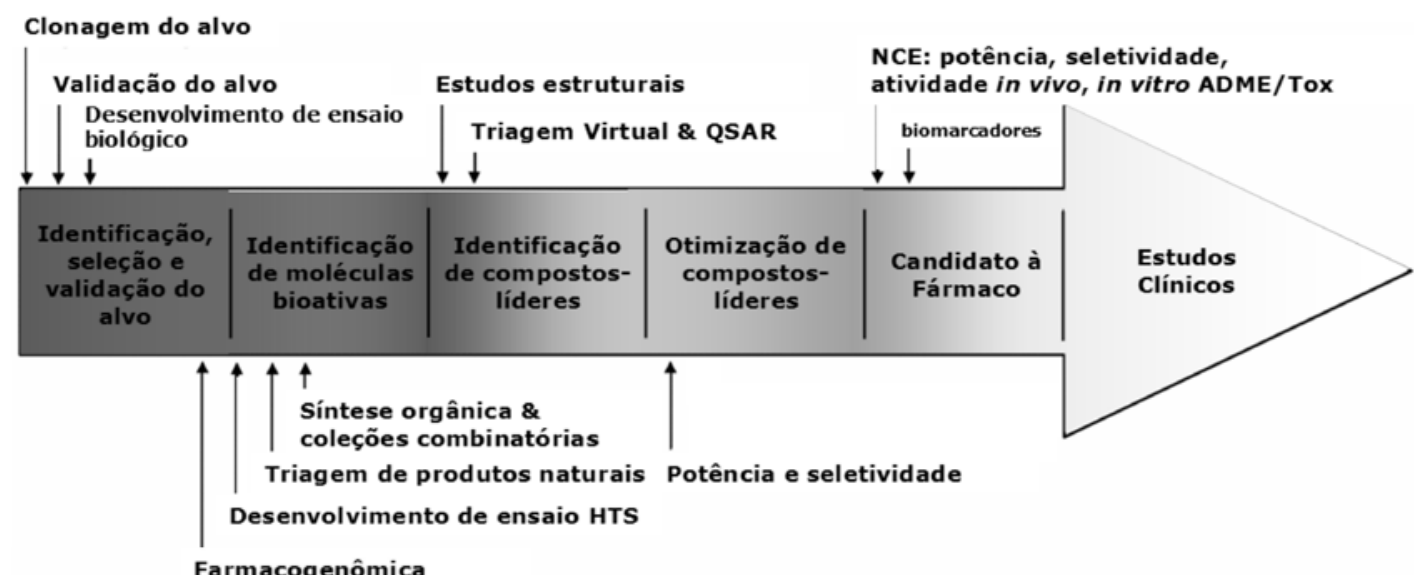

Figura 2: Etapas evolutivas de identificação, seleção e otimização de moléculas no processo de planejamento de fármacos (HTS, triagem em larga escala; QSAR, estudo das relações quantitativas entre a estrutura e atividade; ADME/Tox, propriedades farmacocinéticas/ tóxicas: absorção, distribuição, metabolismo e excreção). ${ }^{24}$

Com o auxílio de métodos em química medicinal é possível explorar o imenso espaço químico delineando o trabalho investigativo na identificação, seleção e otimização de moléculas capazes de interagir com alta afinidade e seletividade com o alvo molecular selecionado (e.g., enzima, receptor), o qual representa o espaço biológico do binômio químico-biológico em questão. Várias estratégias podem ser empregadas, como a organização de bases padrões de dados, a aplicação de filtros moleculares, o emprego de triagens biológicas automatizadas em larga escala (HTS, do inglês, highthroughput screening) e a triagem virtual (VS, do inglês, virtual screening). Além disso, técnicas de planejamento baseado na estrutura do receptor (SBDD, do inglês, structure-based drug design), e de planejamento baseado na estrutura do ligante (LBDD, do inglês, ligand-based drug design) são amplamente utilizadas, bem como o estudo das relações quantitativas entre a estrutura e atividade (QSAR, do inglês, quantitative structure-activity relationships). Em todos estes casos, o gerenciamento qualificado da informação possibilita a geração de conhecimento apropriado a partir dos dados disponíveis. 22,23

Uma das etapas-chave da Figura 2 é a otimização de compostos líderes em relação às suas propriedades farmacodinâmicas e farmacocinéticas, com o objetivo da descoberta de NCEs com elevado potencial de desenvolvimento clínico. Variações estruturais são utilizadas para adequar o perfil das moléculas às características necessárias ao uso terapêutico em humanos. Neste sentido, a química medicinal oferece várias estratégias capazes de gerar coleções dirigidas de compostos. ${ }^{25,26}$ Os estudos de SAR e de QSAR são importantes para guiar a síntese de novas moléculas com propriedades otimizadas, minimizando o universo de compostos a serem considerados nos programas de triagem biológica. Idealmente, propriedades farmacodinâmicas como potência, afinidade e seletividade, além de propriedades farmacocinéticas de ADME (Absorção, Distribuição, Metabolismo e Excreção) devem ser consideradas conjuntamente, facilitando a eliminação de candidatos com propriedades inadequadas e reduzindo os custos do processo de P\&D. ${ }^{27,28} \mathrm{Na}$ fase pré-clínica de pesquisa básica é realizada uma série de testes in vitro e in vivo para avaliar o potencial dos candidatos a NCEs em relação a sua segurança e eficácia, visando a sua introdução em fases clínicas para testes em humanos.

Os métodos computacionais estão integrados ao planejamento de fármacos. O seu emprego nos estudos em química medicinal vão desde a identificação, seleção e otimização de moléculas candidatas até a proposição de novas NCEs para uso clínico. A integração desses métodos ao trabalho químico e biológico experimental é um requerimento essencial para a geração de novas moléculas bioativas qualificadas, com propriedades múltiplas otimizadas. . $^{53,28-30}$ 
Após a conclusão da fase pré-clínica, as NCEs selecionadas são submetidas à aprovação das agências regulatórias, como a Administração Federal de Alimentos e Medicamentos dos Estados Unidos (FDA, do inglês, Food and Drug Administration), para autorização da realização de testes em humanos através da chamada licença para investigação de um novo candidato a fármaco (IND, do inglês, investigational new drug) (Figura 1). No Brasil, a responsável pelo controle das pesquisas clínicas e registro de fármacos é a Agência Nacional de Vigilância Sanitária (ANVISA). Nas fases clínicas são realizados diversos estudos para avaliar a eficácia e a segurança das NCEs candidatas a fármacos. ${ }^{13,16}$

\section{Fundamentos Metodológicos}

\section{ESTRATÉGIAS DE PLANEJAMENTO DE FÁRMACOS}

As estratégias de planejamento de candidatos a novos fármacos fundamentam-se no conhecimento prévio do processo fisiológico envolvido no surgimento da doença alvo e na seleção de um alvo macromolecular adequado. $\mathrm{O}$ alvo molecular (e.g., proteína, DNA ou RNA) pode ter a sua estrutura tridimensional (3D) conhecida ou não, fato que auxilia na escolha das estratégias de planejamento. ${ }^{17}$ Os grandes avanços da genômica e proteômica, bem como a evolução de técnicas como a cristalografia de raios X e ressonância magnética nuclear (RMN), proporcionam um aumento significativo no número de alvos moleculares que possuem suas estruturas 3D disponíveis no Banco de Dados de Proteinas (PDB, do inglês, Protein Data Bank). ${ }^{31,32} \mathrm{O}$ conhecimento da estrutura do alvo macromolecular ou da estrutura de algum complexo ligante-receptor, permite o planejamento de racional de inibidores enzimáticos, ou de agonistas ou antagonistas de receptores através de estratégia de SBDD. ${ }^{1,2,5}$

Em contraste, quando a estrutura do alvo macromolecular eleito não é conhecida, aplicam-se métodos de LBDD envolvendo o estudo de características multifuncionais de ligantes bioativos conhecidos., ${ }^{1,33,34}$ Em muitos casos, o uso integrado das técnicas de SBDD e LBDD pode gerar informações e novos conhecimentos úteis no planejamento de NCEs, explorando a sinergia e complementaridade inerente entre as técnicas. ${ }^{1,35}$

\section{QUÍMICA MEDICINAL E OS MÉTODOS DE MODELAGEM MOLECULAR}

Métodos computacionais são ferramentas amplamente empregadas nos projetos de $\mathrm{P} \& \mathrm{D}$ de novos fármacos, desde a identificação de moléculas com atividade biológica até a otimização múltiplas de propriedades de compostos líderes. Devido à rápida evolução nas áreas de hardware, software e métodos, os processos computacionais anteriormente executados exclusivamente em estações de trabalho de alto desempenho são hoje em dia realizados em microcomputadores numa escala de tempo bastante razoável. Diante desse cenário, a integração entre técnicas experimentais e computacionais assumiu grande importância no processo de planejamento de fármacos. ${ }^{36-}$ ${ }^{38}$ Neste contexto, duas estratégias de grande impacto destacam-se, o SBDD e o LBDD.

\section{PLANEJAMENTO BASEADO NA ESTRUTURA DO RECEPTOR}

O SBDD é uma técnica baseada no conhecimento do arranjo topológico de alvos moleculares, logo, utiliza como pré-requisito a informação $3 \mathrm{D}$ detalhada da macromolécula em estudo. Essa informação é geralmente adquirida através da análise de estruturas obtidas por cristalografia de raios $\mathrm{X}$, estudos de RMN ou modelagem por homologia. ${ }^{1}$ Tais estruturas encontram-se depositadas em bases de dados públicas onde podem ser acessadas livremente. As principais bases de dados são: $\mathrm{PDB},{ }^{31}$ InterPro, ${ }^{39-41}$ ExPASy, ${ }^{42}$ e Relibase. ${ }^{43-45}$

A docagem molecular (do inglês, molecular docking), ou simplesmente docagem ou ancoramento (do inglês, docking), é uma dos principais métodos de SBDD empregados em estudos de química medicinal. Esta técnica consiste na predição da conformação bioativa de uma micromolécula (ligante) no sítio de ligação de uma macromolécula (e.g., enzima, receptor, DNA ou RNA), seguida da avaliação (pontuação) e classificação do modo de ligação proposto. ${ }^{1,36}$ A Figura 3 apresenta um esquema geral do processo de do cagem molecular. Uma estratégia bastante difundida que emprega os métodos de docagem molecular é a triagem virtual (VS). Nesta aplicação, os programas de docagem molecular são utilizados para identificar e classificar as conformações bioativas de moléculas pertencentes a bases de dados podendo conter 
centenas de milhares de compostos disponíveis para aquisição comercial ou síntese. ${ }^{36}$

A docagem molecular foi introduzida no início da década de 80 tendo contribuído significativamente tanto na fase de descoberta de novas moléculas bioativas, quanto na fase de otimização de compostos líderes em relação a uma série de propriedades, como potência e afinidade, além de algumas propriedades farmacocinéticas, entre outras. ${ }^{36,46} \mathrm{Um}$ dos primeiros grandes desafios em SBDD é a seleção do programa de docagem molecular. Os métodos disponíveis são baseados em conceitos que diferem ligeiramente entre si, fazendo com que um programa em particular seja mais adequado para um determinado projeto. É estimado que existam aproximadamente 30 programas de docagem molecular disponíveis, ${ }^{47}$ entre os quais destacam-se o DOCK, ${ }^{48}$ Gold, ${ }^{49}$ FlexX, ${ }^{50}$ Glide,${ }^{51,52}$ Autodock, ${ }^{53}$ e Surflex-Dock. ${ }^{54}$

$\mathrm{O}$ processo de docagem molecular pode ser dividido em duas etapas principais: (i) modelagem do modo de ligação (predição da conformação bioativa) e (ii) predição da afinidade do ligante pelo sítio. A predição do modo de ligação de moléculas no sítio de interação de receptoresalvos é considerada a etapa mais simples e robusta do processo. ${ }^{38}$ Para realizar essa primeira etapa, os programas utilizam diferentes métodos de amostragem que atribuem flexibilidade à molécula do ligante. Os métodos de amostragem podem ser classificados em três categorias principais: (i) Métodos Sistemáticos (e.g., Construção Incremental, Amostragem Conformacional); (ii) Métodos Aleatórios ou Estocásticos (e.g., Método de Monte Carlo, Algoritmo Genético); e (iii) Métodos de Simulação (e.g., Dinâmica Molecular e Minimização de Energia). A etapa de predição da afinidade do ligante pelo sítio de ligação é realizada baseada em funções de pontuação desenvolvidas para avaliar e classificar o modo de interação proposto na etapa anterior. As funções de pontuação utilizadas podem ser agrupadas em três categorias básicas: ${ }^{55}$ (i) Campos de Força, geralmente baseados em métodos de mecânica molecular, quantificam a energia proveniente das interações entre o ligante e receptor e a energia interna do ligante (e.g., G-score e D-score, implementados no pacote de programas SYBYL; $;{ }^{56}$ Amber, ${ }^{57}$ implementado no programa AUTODOCK; ${ }^{53}$ GOLDscore implementado no programa GOLD); ${ }^{49}$ (ii) Empíricas, são funções baseadas no ajuste teórico de dados experimentais através do emprego de equações (e.g., regressão linear) (e.g., LigScore, ${ }^{58}$ PLP,${ }^{58}$ LUDI,${ }^{58}$ F-Score,${ }^{56}$ ChemScore ${ }^{56}$ e X-score ${ }^{56}$ ); (iii) Funções Baseadas no Conhecimento, que utilizam dados estatísticos dos potenciais de pares atômicos de interação, os quais são derivados de conjuntos de dados proveniente de complexos cristalográficos entre proteínas e ligantes (e.g., $\mathrm{PMF}^{58}$ e DrugScore ${ }^{60}$ ).

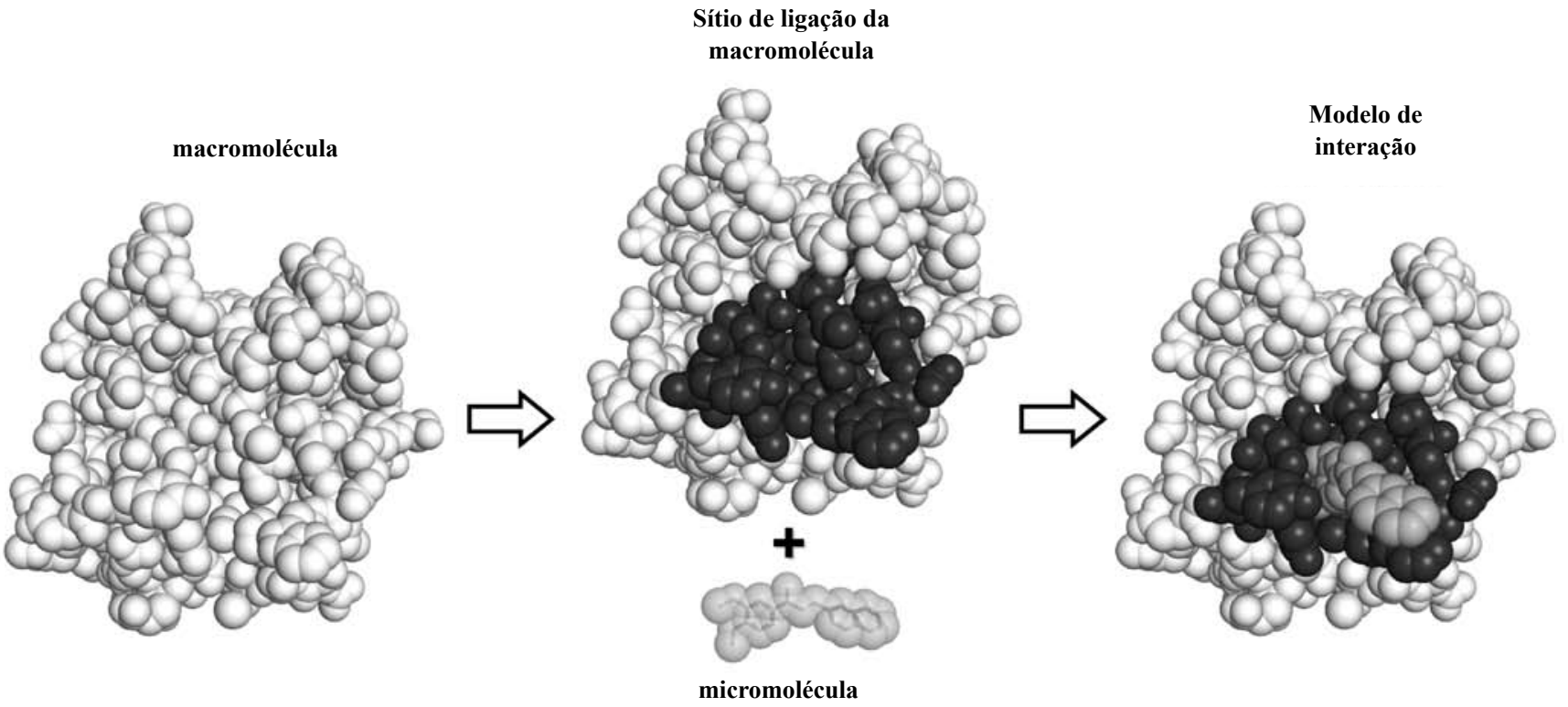

Figura 3: Etapas envolvidas no processo de docagem molecular. 
No processo de reconhecimento molecular, a energia livre de ligação (Energia livre de Gibbs) é determinada pela combinação das contribuições entálpicas e entrópicas. ${ }^{38}$ Esse conceito é implementado nas funções de pontuação de maneira aproximada, uma vez que o componente entálpico está razoavelmente caracterizado e descrito, entretanto, as contribuições entrópicas são difíceis de descrever, pois ainda não são totalmente compreendidas e dependem de um número significativo de fatores, como, por exemplo, a área de superfície hidrofóbica, a liberação de moléculas de água ligadas à cavidade de ligação (dessolvatação) e a imobilização das ligações rotacionáveis na molécula do ligante $^{38}$. Portanto, as funções de pontuação implementadas nos programas de docagem molecular são funções simplificadas e aproximadas. Entretanto, apesar de explicarem parcialmente o processo de interação ligantemacromolécula, são úteis nos processos de descoberta de novos compostos com atividade biológica.

\section{PLANEJAMENTO BASEADO NA ESTRUTURA DO LIGANTE}

Os métodos de LBDD, muito empregados na identificação de novas moléculas bioativas e na otimização de compostos líderes, não exigem informação sobre a topologia do alvo molecular. ${ }^{61,62}$ As principais estratégias de LBDD são baseadas na análise comparativa de moléculas bioativas e podem ser classificadas em quatro categorias principais: (i) Buscas por Similaridade, que utilizam principalmente métodos de impressão digital molecular (do inglês, molecular fingerprint) para identificar e classificar compostos como similares ou diferentes de um ligante bioativo. ${ }^{63}$ Esses métodos são baseados no princípio das propriedades similares, ou seja, moléculas estruturalmente relacionadas podem apresentar atividade biológica similar; (ii) Farmacóforos, comumente definidos como um arranjo tridimensional de propriedades moleculares que determinam um conjunto mínimo de condições necessárias para o reconhecimento molecular. Esses métodos envolvem a identificação de padrões farmacofóricos comuns entre conjuntos de moléculas bioativas e a utilização deste padrão (modelo farmacofórico) para buscas de novas moléculas que satisfação os requisitos impostos pelo modelo; ${ }^{64}$ (iii) Inteligência Artificial, técnica que se baseia em regras classificatórias derivadas a partir de um conjunto de moléculas ativas e inativas utilizado para construir e treinar modelos computacionais com a aplicação de técnicas de aprendizado de máquina (do inglês, machine learning). Posteriormente, o modelo desenvolvido é aplicado para a identificação e seleção de novos compostos com atividade biológica; ${ }^{65}$ (iv) Métodos de QSAR, que buscam identificar e quantificar as relações predominantes entre a estrutura química e atividade biológica de um conjunto treinamento de moléculas quimicamente relacionadas através do uso de descritores moleculares especializados. ${ }^{35}$ Os métodos de QSAR (e.g., QSAR 2D, QSAR 3D) representam uma das estratégias de LBDD mais empregadas e têm como objetivo o planejamento de moléculas com propriedades superiores em relação àquelas apresentadas pelo conjunto de dados que deu origem ao processo de modelagem. Para o estabelecimento de estudos com alto padrão de qualidade, as moléculas do conjunto treinamento precisam ser quimicamente relacionadas (ocupar o mesmo espaço químico), exercer seus efeitos em um mesmo alvo terapêutico, atuando na mesma cavidade de ligação e através do mesmo mecanismo de ação. ${ }^{66,67}$ A propriedade alvo, geralmente potência ou afinidade, deve ser padronizada e validada para ser útil no desenvolvimento de modelos quantitativos. Propriedades moleculares (e.g., campos moleculares estereoquímico, eletrostático, hidrofóbico) são calculadas e empregadas como descritores. Métodos estatísticos como a regressão por mínimos quadrados parciais (PLS, do inglês, partial least squares) ou a regressão linear multivariada (MLR, do inglês, multiple linear regression) são usados para produzir uma relação (equação) que descreve as variações na propriedade alvo em função dos descritores moleculares. O processo cíclico de formulação de QSAR, predição da propriedade alvo, síntese e ensaios biológicos, prossegue até que novas moléculas candidatas a NCEs sejam selecionadas com elevado potencial de desenvolvimento clínico.

Os métodos de QSAR são muito explorados em conjunto com métodos de SBDD quando estruturas 3D dos alvos macromoleculares eleitos são disponíveis. ${ }^{1}$ Nesse contexto se destacam os métodos de (i) análise comparativa de campos moleculares (CoMFA, do inglês, comparative molecular field analysis), ${ }^{68} \mathrm{e}$ (ii) análise 
comparativa dos índices de similaridade molecular (CoMSIA, do inglês, comparative molecular similarity indices analysis), que se baseiam na premissa de que a atividade biológica está diretamente relacionada com as interações do tipo fármaco-receptor, ou seja, as moléculas bioativas geralmente produzem seus efeitos através de interações (e.g., de natureza não-covalente) estereoquímicas, eletrostáticas, hidrofóbicas e ligação de hidrogênio com o alvo macromolecular. Como essas interações apresentam caráter tridimensional, estes métodos consideram as moléculas em três dimensões, utilizando campos moleculares de interação (MIFs, do inglês, molecular interaction fields) como descritores moleculares.

\section{MODELAGEM MOLECULAR E O PLANEJAMENTO DE INIBIDORES DE PROTEÍNAS QUINASES}

As proteínas quinases são conhecidas por regular a grande maioria das vias celulares. O genoma humano contém, aproximadamente, 500 genes para proteínas quinases, os quais representam aproximadamente $2 \%$ de todos os genes de eucariotos. ${ }^{70}$ A família das proteínas quinases compreende duas grandes subfamílias: (i) proteínas tirosina quinases e (ii) proteínas serinatreonina quinases. Dentre os membros da subfamília de proteínas serina-treonina quinases, encontramse as Aurora quinases. ${ }^{71}$ Os mamíferos expressam três tipos de Aurora quinases: A, B e C, cujas funções biológicas estão relacionadas à regulação da mitose. $\mathrm{A}$ expressão e atividade quinase das proteínas Aurora A e B são determinadas pelo ciclo celular, de forma que somente durante a mitose estas proteínas são expressas e apresentam atividade biológica. ${ }^{72} \mathrm{~A}$ amplificação e super expressão do gene Aurora A foi observada em diversas linhagens de células tumorais (e.g., mama, ovários, cólon, próstata, neuroblastoma e cervical), sugerindo que a proteína Aurora A estaria relacionada à transformação oncogênica. ${ }^{73}$ Estes resultados levaram a formulação da hipótese de que a inibição da atividade quinase das enzimas Aurora A e B pudesse ser útil no tratamento do câncer. ${ }^{74} \mathrm{O}$ desenvolvimento de inibidores de quinases, como o Imatinib (Gleevec ${ }^{\circledR}$, da Novartis), Gefitinib (Iressa ${ }^{\circledR}$, da AstraZeneca) e Erlotinib (Tarceva ${ }^{\circledR}$, da Genentech/OSIP) ilustram o potencial terapêutico dessa classe de enzimas. Mais recentemente, um potente inibidor de Aurora A e B que se encontra em testes clínicos, o VX-680 (Vertex Pharmaceutical \& Merck), ${ }^{75}$ foi capaz de suprimir o crescimento de tumores in vivo.

Com o objetivo de descobrir novas moléculas inibidoras de Aurora quinases, uma coleção de cerca de 250.000 compostos da AstraZeneca foi testada para se avaliar a capacidade de inibição da enzima Aurora A quinase. ${ }^{76}$ Dentre os compostos testados, a anilinoquinazolina (composto 1, Tabela 2) foi identificada como sendo uma molécula bioativa, passando para os estágios seguintes de desenvolvimento. ${ }^{71}$ Esse composto apresentou excelentes níveis de inibição da Aurora A $\left(\mathrm{IC}_{50}=393 \mathrm{nM}\right)$.

Tabela 2: SAR dos inibidores de Aurora A derivados quinazolina.

\begin{tabular}{ccccc}
\hline & \\
\hline
\end{tabular}

Estudos de SAR envolvendo o grupo quinazolina resultaram na descoberta do composto $2\left(\mathrm{IC}_{50}=0,11\right.$ $\mu \mathrm{M})$, onde o substituinte metoxila da posição C7 do anel quinazolina foi substituído por 3-(1-morfolino) propoxila, sendo este composto, aproximadamente, quatro vezes mais potente que o composto 1 . A avaliação do composto 2 contra a Aurora B demonstrou que este possui boa potência inibitória $\left(\mathrm{IC}_{50}=0,13 \mu \mathrm{M}\right)$, ao passo que o mesmo efeito não foi observado para outras quinases. Estes resultados sugeriram que os derivados de quinazolina são inibidores potentes e seletivos da Aurora quinase A. Apesar de potente e seletivo, o composto 2 não apresentava características farmacocinéticas adequadas para um novo fármaco, possuindo alta lipofilicidade, baixa solubilidade em água e alta extensão de ligação às proteínas plasmáticas. Diante disso, procurou-se diminuir o valor de $\log \mathrm{D}$ (coeficiente de partição octanol/solução 
tamponada $\mathrm{pH} 7,4$ ) através da substituição do grupo anilina por um heterocíclico como a pirimidina. Estratégia semelhante foi utilizada anteriormente para aumentar a solubilidade e polaridade do agente antifúngico tioconazol (Tralen ${ }^{\circledR}$, da Pfizer), administrado por via tópica. A introdução de um substituinte polar $(\mathrm{OH})$ e a substituição dos anéis heterocíclicos (imidazol e tiofeno) por sistemas mais polares (dois anéis triazol), levou a obtenção do fármaco fluconazol (Zoltec $\AA$, da Pfizer), administrado por via oral, que apresenta maior solubilidade e atividade contra as infecções sistêmicas. ${ }^{77}$

$\mathrm{O}$ derivado 5-pirimidina (composto 3, Tabela 2) apresentou menor lipofilia $(\log \mathrm{D}=2,7)$ e aumento da fração livre no plasma $(4,5 \%$ livre $)$, apesar da solubilidade em solução aquosa continuar baixa. Além disso, a modificação estrutural que levou a obtenção do composto 3 teve como reflexo o aumento da potência inibitória $\left(\mathrm{IC}_{50}=3,0 \mathrm{nM}\right.$, Tabela 2). O isômero estrutural 2-pirimidina (composto 4) também foi sintetizado e avaliado frente à enzima Aurora A. Curiosamente, mostrou-se aproximadamente 200 vezes menos ativo que o isômero 5-pirimidina. Diante deste resultado, os pesquisadores da AstraZeneca utilizaram métodos de SBDD (cristalografia de proteínas e docagem molecular) para identificar os fatores moleculares determinantes relacionados com este dado biológico. A análise do modo de interação do derivado 5-pirimidina no sítio catalítico da enzima sugeria que este isômero é mais potente que o isômero 2-pirimidina devido à sua capacidade de formar ligações de hidrogênio adicionais com resíduos do sítio ativo (Figura 4). De acordo com este modo de interação, o isômero 2-pirimidina presente (composto 4) não seria capaz de realizar essas interações (Figura 4), oferecendo assim uma explicação para a menor atividade inibitória deste derivado. Posteriormente, foi obtido um complexo cristalográfico entre o domínio catalítico da Aurora A e derivado de quinazolínico 5-pirimidina (código PDB, 2C6E), que revelou o modo de ligação do composto e comprovou os dados de modelagem molecular.

Utilizando a estrutura de $\mathbf{3}$ como composto líder, foram investigados os efeitos no padrão de substituição do anel fenila do gupo benzamida. ${ }^{71}$ Os resultados

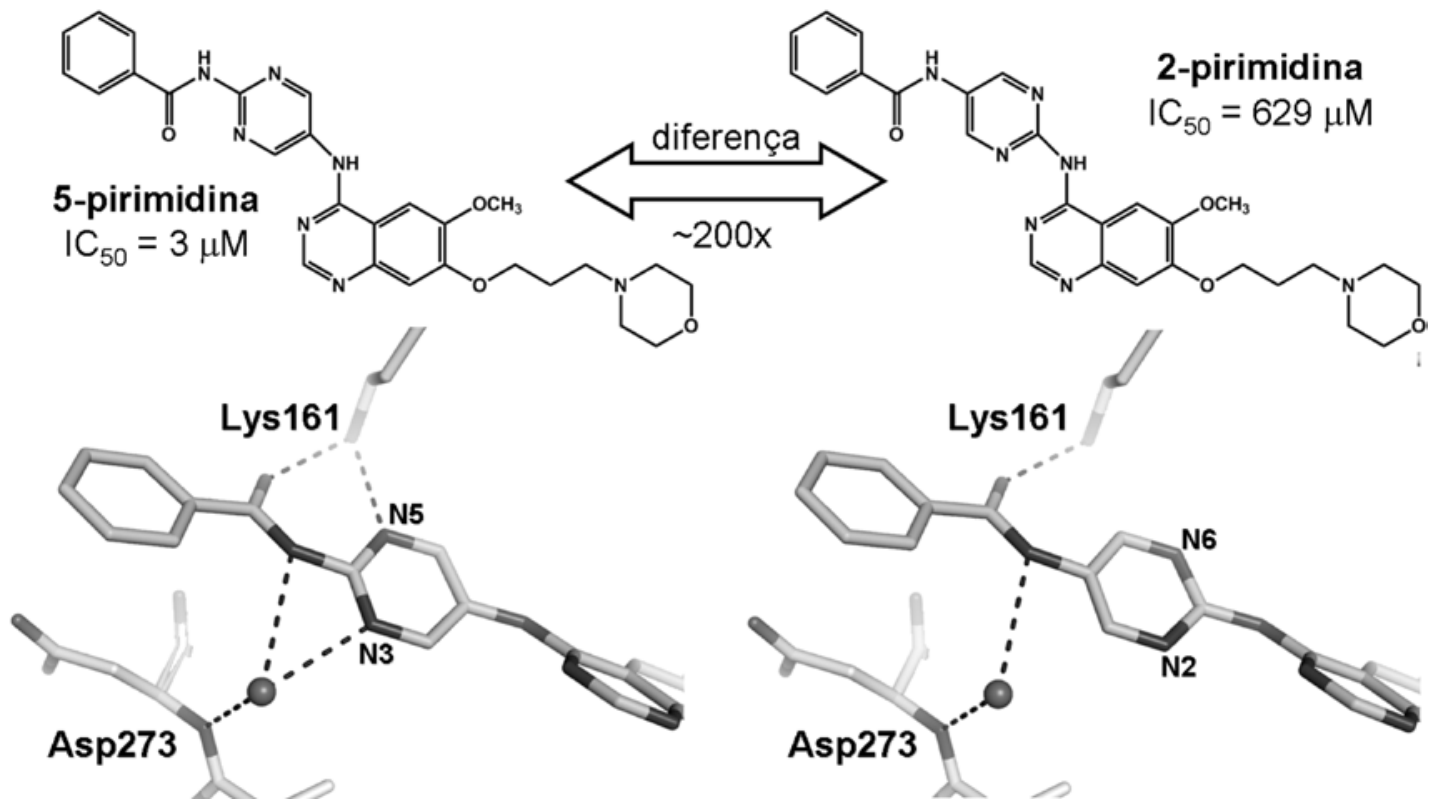

Figura 4. Painel esquerdo: Estrutura molecular e modelo de interação do isômero mais potente 5-pirimidina e os resíduos do sítio ativo da Aurora A. Painel direito. Estrutura molecular e modelo de interação do isômero menos ativo 2-pirimidina e os resíduos do sítio ativo da Aurora A. As moléculas dos inibidores e dos resíduos do sítio de ligação estão representadas em modelos bastão, a molécula de água está representada em modelo de esfera e as ligações de hidrogênio estão representadas como linhas tracejadas. O isômero 5-pirimidina estabelece duas ligações de hidrogênio adicionais quando comparado com o isômero 2-pirimidina: i) entre o N3 do anel pirimidina com uma molécula de água, a qual está interagindo com o NH da cadeia principal do Asp273, ii) entre o N5 do anel pirimidina com o Nz da cadeia lateral da Lys161. 
indicavam uma preferência por substituintes pequenos e lipofílicos, por exemplo, os análogos 3-cloro e 3-cloro4-flúor (compostos 5 e 6, Tabela 3). Estes compostos apresentaram elevada potência in vitro contra a Aurora A, bem como promissora atividade celular $(80$ e $20 \mathrm{nM}$, respectivamente). A introdução de substituintes maiores (compostos 7 e 8) foi desfavorável, enquanto que a introdução do substituinte sulfonilamina (composto 9) resultou numa diminuição de 1.000 vezes na potência biológica quando comparada com o composto 3 (Tabela 3). A substituição do anel fenila por um substituinte heterocíclico (4-piridil, composto 10) ou alquílico (n-butila, composto 11) levou a moléculas com maior solubilidade em água, entretanto, com potência reduzida. Apesar de melhorar a potência, a introdução de halogênios no anel fenila resultou num aumento da lipofilia, fato que determinou uma redução na solubilidade e uma maior ligação às proteínas plasmáticas desses compostos.

Estudos de modelagem molecular indicaram que o grupo morfolino do $\mathrm{C} 7$ da quinazolina estaria posicionado numa região acessível ao solvente. $\mathrm{O}$ refinamento
Tabela 3: SAR dos inibidores de Aurora A, substituições no anel benzamida.<smiles>COc1cc2c(Nc3cnc(NC=O)nc3)ncnc2cc1OCCCN1CCOCC1</smiles>

\begin{tabular}{clr} 
Composto & \multicolumn{1}{c}{ X } & IC $_{\mathbf{5 0}}(\mathbf{n M})$ \\
\hline $\mathbf{5}$ & 3-clorofenila & $<0,10$ \\
$\mathbf{6}$ & 3-cloro-4-fluorfenila & 0,15 \\
$\mathbf{7}$ & 3-bromo-4-metilfenila & 70 \\
$\mathbf{8}$ & 4-etilfenila & 85 \\
$\mathbf{9}$ & (4-dipropilaminosulfonil)fenila & 3.900 \\
$\mathbf{1 0}$ & 4-piridil & 690 \\
$\mathbf{1 1}$ & n-butila & 17 \\
\hline
\end{tabular}

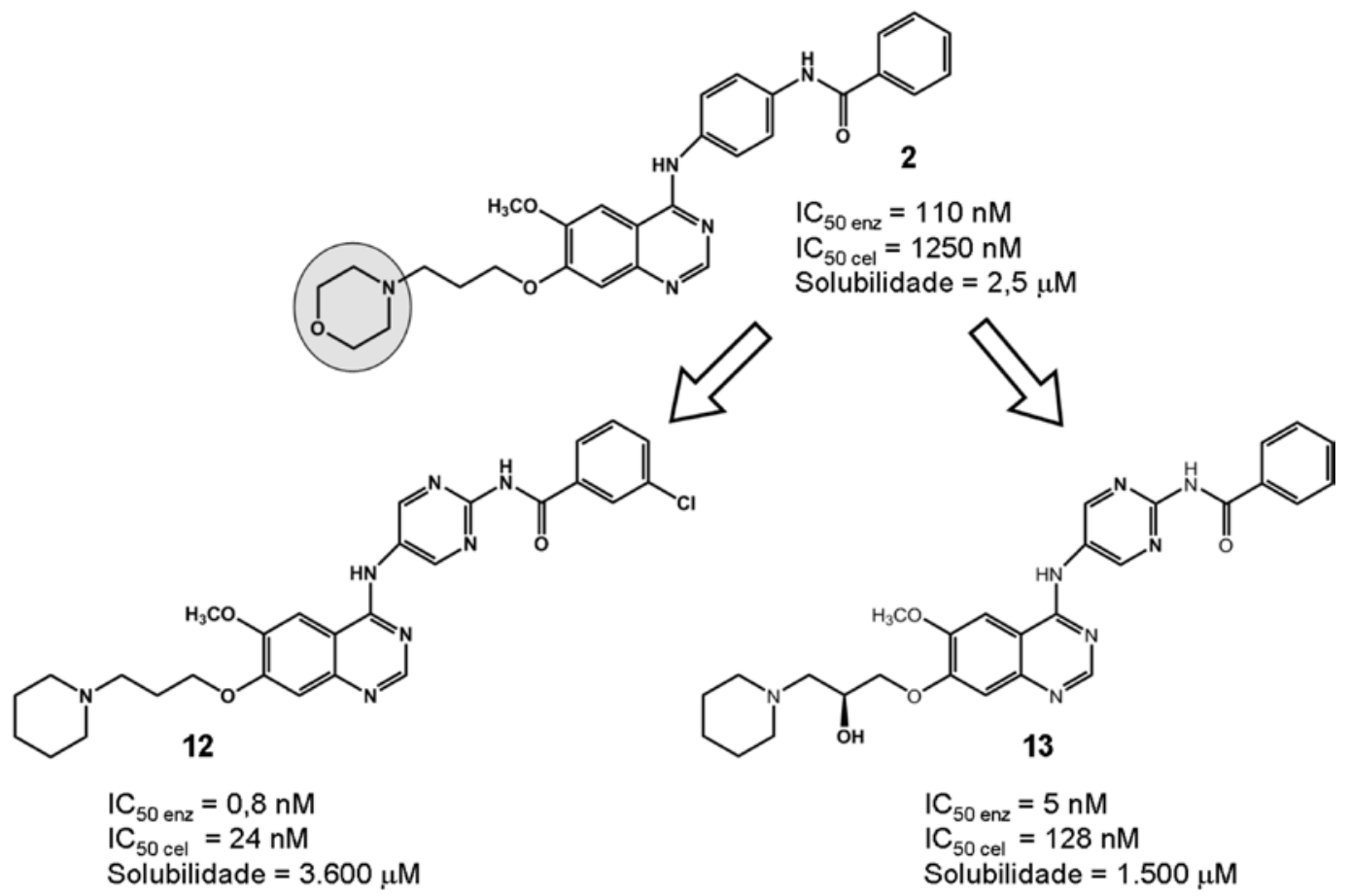

Figura 5: Composto líder (2) e candidatos a fármacos na terapia do câncer (12 e 13). 
posterior do modelo sugeriu a introdução de grupos que pudessem causar um amento da solubilidade. Baseado neste modelo, a substituição do grupo morfolino por piperidino foi capaz de aumentar significativamente a solubilidade e água (composto 12). Embora altere a potência inibitória dessa molécula contra a Aurora A nos ensaios enzimático $\left(\mathrm{IC}_{50}=0,8 \mathrm{nM}\right)$ e celular $\left(\mathrm{IC}_{50}\right.$ $=24 \mathrm{nM}$ ) quando comparada aos demais análogos, essa modificação molecular resultou em uma potência aceitável, tornando o composto 12 (Figura 5) candidato a fármaco na terapia do câncer. Diante desse resultado, os pesquisadores da AstraZeneca ainda retiraram o substituinte cloro do anel fenila e adicionaram um grupo hidroxila na cadeia alifática entre o $\mathrm{C} 7$ da quinazolina e o grupo piperidino (composto 13, Figura 5). O composto 13 apresentou boa solubilidade em água e potência nos ensaios enzimático e celular $\left(\mathrm{IC}_{50}=5 \mathrm{nM}\right.$ e $128 \mathrm{nM}$, respectivamente). Atualmente, o composto 13 encontrase em fase clínica de desenvolvimento. ${ }^{78}$

\section{Conclusão}

Os métodos computacionais de modelagem molecular de fármacos desempenham papel extremamente importante em química medicinal moderna e no processo de planejamento de fármacos, tanto na indústria quanto na academia. A criação de modelos de interação intermolecular e de QSAR permite a identificação de características químicas e estruturais essenciais no processo de reconhecimento molecular. Essas informações, aliadas ao conhecimento em química medicinal e química sintética, são fundamentais na geração de novas classes de candidatos a fármacos com propriedades farmacodinâmicas e/ou farmacocinéticas otimizadas.

\section{REFERÊNCIAS}

1. Guido, R. V. C.; Oliva, G.; Andricopulo, A. D. Curr. Med. Chem. 2008, 15, 37.

2. Davis, A. M.; Teague, S. J.; Kleywegt, G. J. Angew. Chem. Int. Ed. Engl. 2003, 42, 2718.

3. Caprino, L.; Russo. P. Drug Discov. Today 2006, 11, 999.

4. Yago, G.; Amram, M.; Magula, T. Drug Discov. Dev.
2006, 12, p. 22.

5. Kitchen, D. B.; Decornez, H.; Furr, J. R.; Bajorath, J. Nat. Rev. Drug Discov. 2004, 3, p. 935.

6. Kuhn, D.; Weskamp, N,; Schmitt, S.; Hüllermeier, E.; Klebe, G. J. Mol. Biol. 2006, 359, 1023.

7. Lombardino, J. G.; Lowe, J. A. 3rd. Nat. Rev. Drug Discov. 2004, 3, 853.

8. Wermuth, C. G. The Practice of Medicinal Chemistry. Academic Press: London, 2003.

9. Agnew, B. Science 2000, 287, 1952.

10. Cohen, F. J. Nat. Rev. Drug Discov. 2005, 4, 78.

11. Couzin, J. Science 2005, 309, 728.

12. Berndt, E. R.; Gottschalk, A. H.; Philipson, T. J.; Strobeck, M. W. Nat. Rev. Drug Discov. 2005, 4, 545.

13. Dimasi, J. A.; Hansen, R. W.; Grabowski, H. G. J. Health Econom. 2003, 22, 151.

14. United States Government Accountability Office (2006). New drug development. Science, business, regulatory, and intellectual property issue cited as hampering drug development efforts. Disponível em: $<$ http://www.gao.gov/new.items/d0749.pdf $>$. Acesso em: 20 Nov 2008.

15. Medical Marketing and Media. Top 20 pharma companies. Disponível em: <http://www.mmmonline.com/. Acesso em: 20 Nov 2008.

16. McGee, P. Drug Discov. Dev. 2006, 9, 16.

17. Klebe, G. Drug Discov. Today 2006, 11, 580.

18. Anders, H. J.; Vielhauer, V. Drug Discov. Today 2007, $12,446$.

19. Salum, L. B. Estudos in silico no planejamento de candidatos a novos fármacos na terapia do câncer de mama e de reposição hormonal. 2007. $140 \mathrm{f}$. Dissertação (Mestrado em Física Biomolecular) Instituto de Física de São Carlos, Universidade de São Paulo, São Carlos, 2007.

20. Barreiro, E. J.; Fraga, C. A. M. Química medicinal: as bases moleculares da ação dos fármacos. Artmed 
Editora: Porto Alegre, 2001.

21. Oprea, T. I.; Allu, T. K.; Fará, D. C.; Rad, R. F.; Ostopovici, L.; Bologa, C. G. J. Comput. Aided Mol. Des. 2007, 21, 113.

22. Thomas, G. Fundamentals of medicinal chemistry. Wiley:West Sussex, 2003.

23. Jónsdóttir S. O.; Jorgensen, F. S.; Brunak, S. Bioinformatics 2005, 21, 2145.

24. Belfield, G. P.; Delaney, S. J. Biochem. Soc. Trans. 2006, 34, 313.

25. Kogej, T.; Engkvist, O.; Blomberg, N.; Muresan, S. J. Chem. Inf. Model. 2006, 46, 1201.

26. Balakin, K. V.; Tkachenko, S. E.; Kiselyov, A. S.; Savchuk, N. P. Drug Discov. Today: Technol. 2006, 3, 397 .

27. Bunin, A. B. Drug. Discov. Today 2003, 18, 823.

28. Andricopulo, A. D.; Montanari, C. A. Mini Rev. Med. Chem. 2005, 5, 585.

29. Holbrook, J. D.; Sanseau, P. Drug Discov. Today 2007, 12, 826.

30. Russo, E. Nature 2002, 419, 4.

31. Berman, H. M.; Bhat, T. N.; Bourne, P. E.; Feng, Z.; Gilliland, G.; Weissig, H.; Westbrook, J. Nat. Struct. Biol. 2000, Suppl, 957.

32. Westbrook, J.; Feng, Z.; Chen, L.; Yang, H.; Berman, H. M. Nucleic Acids Res. 2003, 31, 489.

33. Eckert, H.; Bajorath, J. Drug Discov. Today 2007, 12, 225.

34. Willett, P. Drug Discov. Today 2006, 11, 1046.

35. Kubiniy, H. 3D QSAR in drug design: theory, methods and applications. Kluwer Academic: Leiden, 1993. v. 1 .

36. Moitessier, N.; Englebienne, P.; Lee, D.; Lawandi, J.; Corbeil, C. R. Br. J. Pharmacol. 2008, 153, S7.

37. Davis, A. M.; St-Gallay, S. A.; Kleywegt, G. J. Drug Discov. Today 2008, 13, 831.

38. Sams-Dodd, F. Drug Discov. Today, 12, 2007.
39. Zdobnov, E. M.; Apweiler, R. Bioinformatics 2001, 17,847 .

40. Mulder, N. J.; Apweiler, R.; Attwood, T. K.; Bairoch, A.; Bateman, A. Nucleic Acids Res. 2007, 35, D2248.

41. Mulder, N. J.; Apweiler, R.; Attwood, T. K.; Bairoch, A.; Bateman, A. Nucleic Acids Res. 2005, 33, D201.

42. Gasteiger, E.; Gattiker, A.; Hoogland, C.; Ivanyi, I.; Appel, R. D.; Bairoch, A. Nucleic Acids Res. 2003, 31, 3784.

43. Hendlich, M.; Bergner, A.; Günther, J.; Klebe, G. J. Mol. Biol. 2003, 326, 607.

44. Günther, J.; Bergner, A.; Hendlich, M.; Klebe, G. J. Mol. Biol. 2003, 326, 621.

45. Bergner, A. Günther, J.; Hendlich, M.; Klebe, G.; Verdonk, M. Biopolymers 2002, 61, 99.

46. Kuntz, I. D.; Blaney, J. M.; Oatley, S. J.; Langridge, R.; Ferrin, T. E. J. Mol. Biol. 1982, 161, 269.

47. Leach, A. R.; Shoichet, B. K.; Peishoff, C. E. J. Med. Chem. 2006, 49, 5851.

48. Shoichet, B.K.; Kuntz, I.D. J. Mol. Biol. 1991, 22, 327.

49. Jones, G.; Willett, P.; Glen, R. C.; Leach, A. R.; Taylor, R. J. Mol. Biol. 1997, 267, 727.

50. Rarey, M.; Kramer, B.; Lengauer, T.; Klebe, G. J. Mol. Biol. 1996, 261, 470.

51. Friesner, R. A.; Banks, J. L.; Murphy, R. B.; Halgren, T. A.; Klicic, J. J.; Mainz, D. T.; Repasky, M. P.; Knoll, E. H.; Shelley, M.; Perry, J. K.; Shaw, D. E.; Francis, P.; Shenkin, P. S. J. Med. Chem. 2004, 47, 1739.

52. Halgren, T. A. ; Murphy, R. B;, Friesner, R. A.; Beard, H. S.; Frye, L. L.; Pollard, W. T.; Banks, J. L. J. Med. Chem. 2004, 47, 1750.

53. Morris, G. M.; Goodsell, D. S.; Halliday, R. S.; Huey, R.; Hart, W. E.; Belew, R. K.; Olson, A. J. J. Comput. Chem. 1998, 19, 1639.

54. Jain, A. N. J. Med. Chem. 2003, 46, 499.

55. Wang, R.; Lu, Y.; Wang, S. J. Med. Chem. 2003, 46, 
2287.

56. Tripos - Sybyl, 2008. Disponível em: <http://www. tripos.com/>. Acessado em: $12 \mathrm{Fev} 2008$.

57. Weiner, S. J.; Kollman, P. A.; Nguyen, D. T.; Case, D. A. J. Comput. Chem. 1986, 7, 230.

58. Accelerys - cerius2, 2008. Disponível em: http:// www.accelrys.com/products/cerius2/>. Acessado em: 12 Fev 2008.

59. Wang, R.; Lai, L.; Wang, S. J. Comput.-Aided Mol. Des. 2002, 16, 11.

60. Gohlke, H.; Hendlich, M.; Klebe, G. J. Mol. Biol. 2000, 295, 337.

61. Lengauer, T.; Lemmen, C.; Rarey, M.; Zimmermann, M. Drug Discov. Today 2004, 9, 27.

62. Eckert, H.; Bajorath, J. Methods Mol Biol. 2008, 453, 349.

63. Willett, P. Drug Discov. Today 2006, 11, 1046.

64. Mason, J. S.; Good, A. C.; Martin, E. J. Curr. Pharm. Des. 2001, 7, 567.

65. Warmuth, M. K.; Liao, J.; Rätsch, G.; Mathieson, M.; Putta, S.; Lemmen, C. J. Chem. Inf. Comput. Sci. 2003, 43, 667.

66. Guido R. V. C.; Trossini, G. H.; Castilho, M. S.; Oliva, G.; Ferreira, E. I.; Andricopulo, A. D. J. Enz. Inhib. Med. Chem. 2008, 23, 964.

67. Guido, R. V. C.; Castilho, M. S.; Mota, S. G: R.; Oliva, G.; Andricopulo, A. D. QSAR Comb. Sci., 2008, 27, 768.

68. Cramer, R. D. ; Patterson, D. E.; Bunce, J. D. J. Am. Chem. Soc. 1988, 110, 5959.

69. Klebe, G.; Abraham, U.; Mietzner, T. J. Med. Chem. 1994, 37, 4130 .

70. Scapin G., Drug Discov. Today 2002, 7, 601.

71. Heron, N. M.; Anderson, M.; Blowers, D. P.; Breed, J.; Eden J. M.; Green, S.; Hill, G. B.; Johnson, T.; Jung, F. H.; McMiken, H. H.; Mortlock, A. A.; Pannifer, A. D.; Pauptit, R. A.; Pink, J.; Roberts, N. J.; Rowsell, S., Bioorg. Med. Chem. Lett. 2006, 16, 1320.
72. Jung, F. H.; Pasquet, G.; Lambert-van der Brempt, C.; Lohmann, J. J.; Warin, N.; Renaud, F.; Germain, H.; De Savi, C.; Roberts, N.; Johnson, T.; Dousson, C.; Hill, G. B.; Mortlock, A. A.; Heron, N.; Wilkinson, R. W.; Wedge, S. R.; Heaton, S. P.; Odedra, R.; Keen, N. J.; Green, S.; Brown, E.; Thompson, K.; Brightwell, S. J. Med. Chem. 2006, 49, 955.

73. Nowakowski, J.; Cronin, C. N.; McRee, D. E.; Knuth, M. W.; Nelson, C. G.; Pavletich, N. P.; Rogers, J.; Sang, B. C.; Scheibe, D. N.; Swanson, R. V.; Thompson, D. A. Structure 2002, 12, 1659.

74. Keen, N.; Taylor, S. Nat Rev Cancer 2004, 12, 927.

75. Harrington, E. A.; Bebbington, D.; Moore, J.; Rasmussen, R. K.; Ajose-Adeogun, A. O.; Nakayama, T.; Graham, J. A.; Demur, C.; Hercend, T.; DiuHercend, A.; Su, M.; Golec, J. M.; Miller, K. M.; Nat. Med. 2004, 10, 262.

76. Ditchfield, C.; Johnson, V. L.; Tighe, A.; Ellston, R.; Haworth, C.; Johnson, T.; Mortlock, A.; Keen, N.; Taylor, S. S. J. Cell. Biol. 2003, 161, 267.

77. Patrick, G. L., Oxford: University Press, 2o ed, 2001.

78. Mortlock, A. A.; Keen, N. J.; Jung, F. H.; Heron, N. M.; Foote, K. M.; Wilkinson, R. W.; Green, S. Curr. Top. Med. Chem. 2005, 5, 807.

\section{Rafael V. C. Guido*1 \& Adriano D. Andricopulo'}

${ }^{1}$ Laboratório de Química Medicinal e Computacional, Centro de Biotecnologia Molecular Estrutural, Instituto de Física de São Carlos, Universidade de São Paulo, Av. Trabalhador São-Carlense 400, 13560-970, São Carlos - SP, Brazil.

"E-mail: rvcguido@ifsc.usp.br 